\title{
New editors take over
}

\author{
Klaas R. Westerterp · Håkan Westerblad
}

Received: 18 February 2013/Accepted: 25 February 2013/Published online: 8 March 2013

(c) Springer-Verlag Berlin Heidelberg 2013

Taking on the role of new Editors-in-Chief (EiC) of European Journal of Applied Physiology (EJAP), a journal with a broad view on physiology, is a challenge. Especially when taking over from the previous EiC, Susan Ward, who has done a remarkable job with the journal. EJAP under her command, for instance, has maintained a stable ranking among the best journals in the field and shown steadily increasing two-year and five-year impact factors. Thus, to meet the challenge as new $\mathrm{EiC}$, we find it advantageous to share the responsibilities. We are also happy to work with a group of highly qualified Editors and Advisory Editors who together cover all the aspects of applied physiology.

As new EiC, we will continue the work to make the journal highly attractive for readers as well as for authors. To this end, we have initiated some changes:

1. The Abstracts of original articles will be more structured, and hence easier to grasp, by including fixed headings starting with the purpose of the study and ending with a conclusion, which should highlight the physiological relevance of the results.

2. In the Results section, we encourage Figures with experimental records. The best way of illustrating the results of physiological experiments is often to show representative original records of, for instance, important difference(s) between before and after treatment or

Communicated by Klaas Westerterp/Håkan Westerblad.

K. R. Westerterp

University of Maastricht, Maastricht, The Netherlands

e-mail: ejapeditors@mrum.eu

H. Westerblad ( $\square)$

Karolinska Institutet, Stockholm, Sweden

e-mail: ejapeditors@mrum.eu between the population $\mathrm{A}$ and $\mathrm{B}$. Conversely, we discourage inclusion of extensive statistical analyses in the text. The outcome of statistical analyses presented in the text should, in most cases, be restricted to those used to test specified hypotheses. Detailed description of aspects such as the demographics of subjects involved in the study, including statistical analyses, are better presented in tables.

An immediate point of our attention is to reduce the time between the acceptance/online access of publications and their occurrence in the final printed format. EJAP receives an increasing number of submissions and the allocated page numbers cannot be increased at the same rate. To deal with this, the average acceptance rate has to be reduced to about one out of five submissions rather than the earlier one out of three. Priority is then generally given to hypothesisdriven submissions within the aims and scopes of the journal. Submissions that clearly fall outside of this priority will not go through the full reviewing process and authors in this way will get a faster decision.

Future initiatives will be aimed at attracting high-quality papers and ensuring author satisfaction. The result should be a general physiology journal appreciated by the authors and readers who, despite $\mathrm{EJAP}^{\prime}$ s geographical designation, come from all continents. 